\title{
Persistent Left Superior Vena Cava - Accidental Finding
}

\author{
CORNEL SAVU ${ }^{1,2}$, CORNEL PETREANU $^{1,2}$, ALEXANDRU MELINTE $^{1}$, RADU POSEA $^{1}$, IRINA BALESCU $^{3}$, \\ LAURA ILIESCU ${ }^{2,4}$, CAMELIA DIACONU $^{2,5}$, NICOLAE GALIE $^{1,2}$ and NICOLAE BACALBASA ${ }^{2,6,7}$ \\ ${ }^{1}$ Department of Thoracic Surgery, “Marius Nasta” National Institute of Pneumophthisiology, Bucharest, Romania; \\ ${ }^{2}$ Department of Obstetrics and Gynecology, "Carol Davila" \\ University of Medicine and Pharmacy, Bucharest, Romania; \\ ${ }^{3}$ Department of Visceral Surgery, "Ponderas Academic Hospital", Bucharest, Romania; \\ ${ }^{4}$ Department of Internal Medicine, "Fundeni" Clinical Institute, Bucharest, Romania; \\ ${ }^{5}$ Department of Internal Medicine, "Floreasca" Emergency Clinical Hospital, Bucharest, Romania; \\ ${ }^{6}$ Department of Visceral Surgery, Center of Excellence in Translational Medicine, \\ "Fundeni" Clinical Institute, Bucharest, Romania; \\ ${ }^{7}$ Department of Obstetrics and Gynecology, "I. Cantacuzino" Clinical Hospital, Bucharest, Romania
}

\begin{abstract}
Background/Aim: The presence of the superior left vena cava represents a rare anomaly of the thoracic venous system. Case Report: An asymptomatic case of this type of anomaly, discovered as an accident during investigations for a different pathology (superior left pulmonary lobe tumor), is presented. A 56-year-old, heavy smoker was admitted in our clinic with a tumoral mass in the left superior pulmonary lobe discovered during a routine chest x-ray. Physical and clinical examination was normal. However, transthoracic echography noted a coronary sinus enlargement, which led to the suspicion of a thoracic venous anomaly. Contrast chest computed tomography pointed out a venous anomaly at the level of the left hemithorax originating from the cervical region, crossing the aortic arch and draining in the coronary sinus. During the examination, contrast substance was not detected in the right superior vena cava, either early or late during the computed tomography. During surgery the presence of a persistent left superior vena cava was observed, coming from the cervical region, crossing lateral to the aortic arch and draining in the coronary sinus. Conclusion: The presence of an enlarged coronary sinus should warn the surgeon about the possibility of a thoracic venous anomaly. Identifying a persistent left superior vena cava is important due to its clinical
\end{abstract}

This article is freely accessible online.

Correspondence to: Irina Balescu, "Ponderas" Academic Hospital, Bucharest, Romania. Tel: +40 724077709, e-mail: irina.balescu@ ponderas-ah.ro

Key Words: Left superior vena cava, enlarged coronary sinus, anomaly, clinical implications. implications, especially during certain procedures such as mounting central venous lines, cardiac cannulation or implantation of cardiac stimulators.

The persistence of the left superior vena cava (PLSVC) was noted even before 1787 (1), but the first case was described by Marshall in 1850 (2). Starting from 1975, a number of authors (3) have reported the presence of a persistent superior left vena cava in $2.1 \%-4.3 \%$ of patients which also had another congenital cardiac disease $(2,3)$. Even so, the persistence of the left superior vena cava is a less common vascular anomaly while still being the most frequent thoracic venous anomaly. It is found in $0.3-0.5 \%$ of cases, is usually asymptomatic and has no hemodynamic implications. In most cases, persistent left superior vena cava (80-90\%) coexists with the right vena cava; however, in $0.09-0.13 \%$ of cases PLSVC exists in the absence of the right superior vena cava (4). In our case the venous anomaly was discovered accidentally, the patient presented no signs or symptoms, which could suggest this diagnosis.

In this presentation we will point out the clinical implications of this type of venous anomaly, as well as detailing the diagnostic approach and a perspective into the embryologic modifications which lead to this type of anomaly.

\section{Case Report}

A 56-year-old patient was addressed to the Department of Thoracic Surgery, "Marius Nasta" National Institute of Pneumophthisiology for persistent cough and minimal hemoptysis, weight loss (5 kg/30 days), loss of appetite and diffuse thoracic pain. Patient history revealed a surgically treated groin hernia as well as a previous history of heavy 
smoking (-20 pack year). The blood tests revealed normal parameters except an elevated level of fibrinogen as well as an elevated erythrocyte sedimentation rate. The patient was submitted to an anterior chest $\mathrm{x}$-ray which identified a slightly enlarged pulmonary hilum, a $3 / 4 \mathrm{~cm}$ homogenous opacity with no clear margins situated in the subclavian area of the left upper lobe and a bilateral basal bronchiectasis; no other pathological finding was observed.

We went further with the investigation plan and we submitted the patient to bronchoscopy which revealed bilateral bronchitis with no endobronchial proliferative elements; no new information towards the diagnosis was obtained. The thoracic and abdominal CT scan revealed the presence of a $3.5 \mathrm{~cm} / 4.7 \mathrm{~cm}$ mass in the left superior upper lobe with poorly defined margins, in close contact and with possible infiltration of the parietal pleura. No hilar or mediastinal lymph nodes were observed; the abdominal computed tomography excluded the presence of any tumors at the level of the liver or adrenal glands. In the visceral compartment of the mediastinum a vascular structure was observed, with early filling of contrast which appeared to have a point of origin in the upper thoracic region, from the joining of the internal left jugular vein with the left subclavian vein. It should be mentioned that the contrast media was injected through a peripheral vein on the left arm. This large venous structure had a caudal orientation, lateral to the left subclavian artery, going across the aortic arch and draining into the coronary sinus. Further computed tomography images revealed no contrast media in the venous brachiocephalic trunk in association with the presence of the superior vena cava (right), but also with no contrast media reaching it. Therefore, the computed tomography raised the suspicion of the persistence of the left superior vena cava as well as the absence of the venous brachiocephalic trunk (Figure 1).

The patient was further submitted to transthoracic echocardiography which showed an ejection fraction of $55 \%$, preserved function of the left ventricle, normal pressures in the right atrium and a dilated coronary sinus which also pointed to a venous anomaly.

Surgery was performed under general anesthesia with selective intubation. Thoracic access was gained through the $5^{\text {th }}$ left intercostal space by lateral thoracotomy. After opening the thoracic cavity, we observed the presence of the persistent left superior vena cava, entering the thorax through the apex, lateral to the left subclavian artery, descending towards the heart and crossing lateral of the aortic arch. At this level the PLSVC also drained another important venous structure - the left azygos - coming in from the posterior wall and entering the left vena cava near the aortic arch. It continued descending anteriorly to the left pulmonary artery and the left superior pulmonary vein, draining into the coronary sinus (Figure 2). Surgery consisted in left upper lobectomy with mediastinal lymphadenectomy. The histopathological examination confirmed the presence of a pulmonary adenocarcinoma with no mediastinal or hilar lymph node metastasis. The early postoperative evolution was uneventful, the patient being discharged in the seventh postoperative day.

\section{Discussion}

Persistence of the left superior vena cava (PLSVC) can present in two forms depending on where the vessel drains into the heart: in up to $90 \%$ of cases it drains into the coronary sinus, while in up to $10 \%$ of cases it drains directly into the left atrium or one of the pulmonary veins $(5,6)$, in which case a right-to-left shunt may appear. When the venous drainage occurs directly into the coronary sinus, the PLSVC opens directly into the right atrium, in close proximity to the insertion of the atrioventricular valves (7). In $82-90 \%$ of cases PLSVC appears as a duplicated superior vena cava (left and right). In this situation, the innominate vein connecting them is present in $30 \%$ of cases. In most typical cases, in the absence of other cardiovascular malformations, PLSVC does not associate with clinical implications or hemodynamic modifications (8). It has been reported (9) that this type of venous anomaly is more frequently associated with other cardiovascular anomalies such as: pulmonary veins anomalies (in $10 \%$ of cases), pulmonary atresia (in $6 \%$ of cases), tricuspid atresia (in 4$5 \%$ of cases), hypoplasia of the left ventricle (in $2.5 \%$ of cases) or Fallot tetralogy (in $2 \%$ of cases). Among these, arterio-venous fistulas have been described between the left circumflex coronary artery and the great cardiac vein which then drains into the persistent left superior vena cava (10). Others have reported the association of PLSVC with MayThurner syndrome (consisting of compression of the right common iliac vein between the right common iliac artery and the lombar vertebrae) (11).

Isolated or doubled PLSVC (right superior vena cava) may be associated with cardiac defects, such as inter-atrial septal defect; in such cases a right-to-left shunt appears with hemodynamic implications (12). The association between PLSVC and aberrant venous drainages has also been reported, consisting of right subclavian vein draining into the left superior vena cava (13). PLSVC can also be associated with left subclavian vein stenosis and absence of the right superior vena cava (14). Moreover, cases in which PLSVC drains into the left superior pulmonary vein and the presence of usual signs of a right-to-left shunt have been also reported (15).

PLSVC has also been described in association with the absence of the hepatic part of the inferior vena cava (16) and also as part of the Raghib syndrome (PLSVC in association with coronary sinus atresia and atrial septal defect) (17). In 


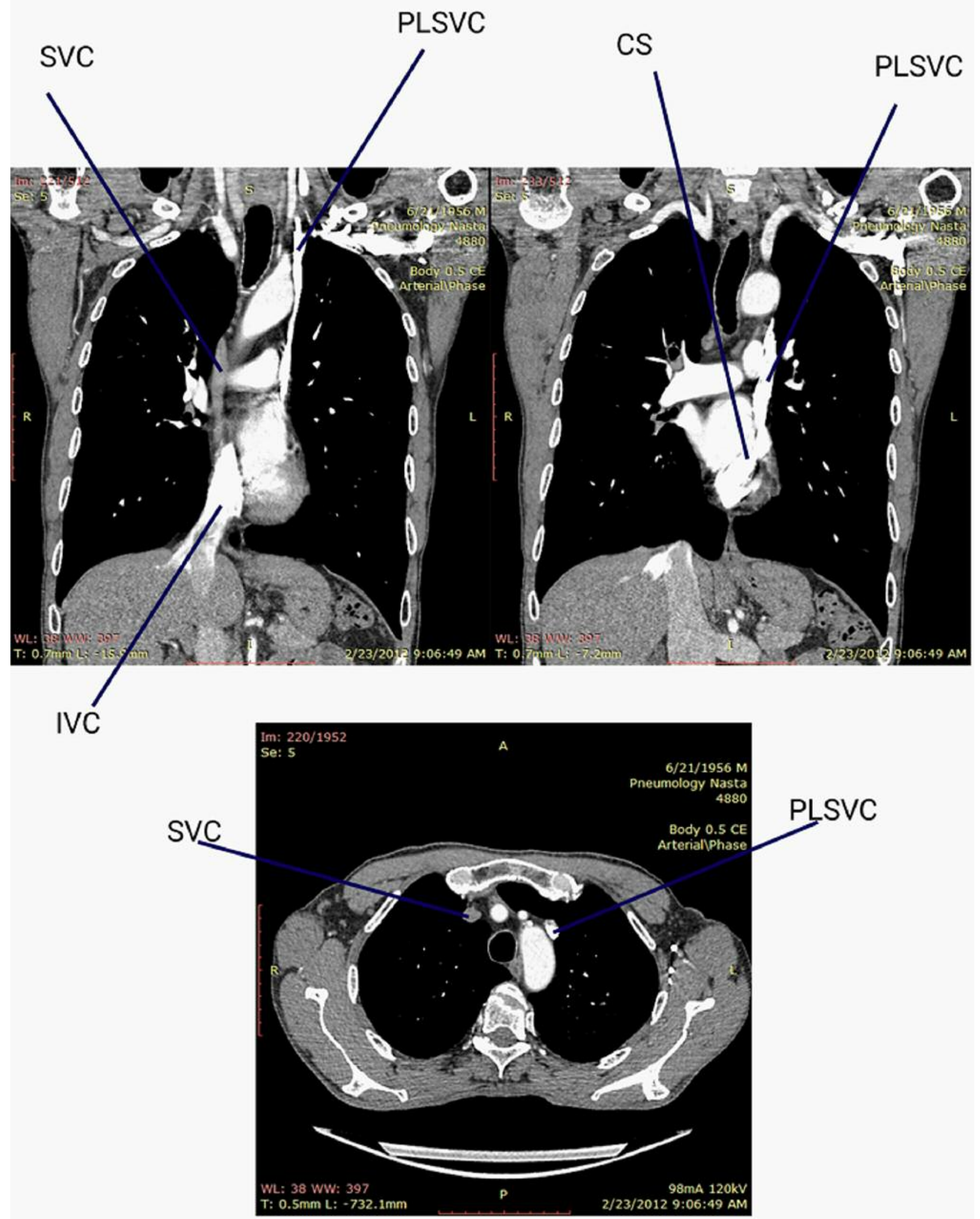

Figure 1. Contrast CT view. SVC: Superior vena cava; PLSVC: persistent left superior vena cava; IVC: inferior vena cava; CS: coronary sinus. 


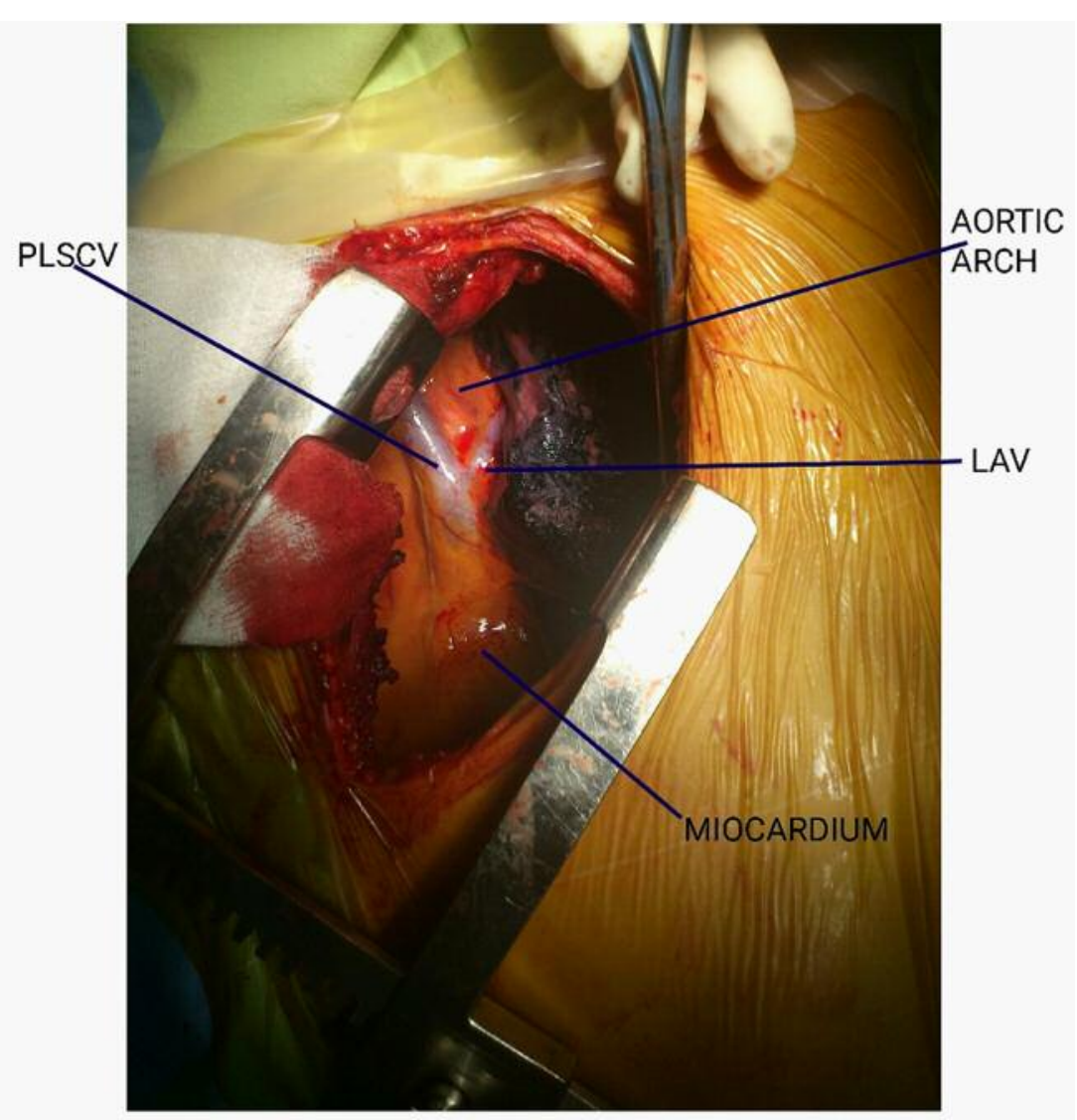

Figure 2. Intraoperative view of PLSCV. PLSVC: Persistent left superior vena cava; LAV: left azygos vein.

other cases, different cardiac arrhythmias may appear along with PLSVC, such as: WPW syndrome or sick sinus syndrome and even sudden death (18). In rare cases, PLSVC is present in complete situs inversus or cardiac dextroposition in association with the absence of the right superior vena cava.

The normal development of the superior vena cava during fetal growth starts in the $4^{\text {th }}$ week of pregnancy, when the anterior and posterior cardinal veins open into the venous sinus through the common cardinal veins. In the $7^{\text {th }}$ week of pregnancy, communication between the anterior left and right cardinal veins leads to the anastomosis of the two segments. This anastomosis will then become the innominate venous trunk. The right superior vena cava forms from the right precardinal and the right common cardinal veins. On the left, the distal portion of the left precardinal vein, beyond the innominate vein anastomosis, atrophies and becomes the Marshalls' ligament, while the cardinal common vein becomes Marshalls' vein - collecting blood from the posterior part of the heart and draining it into the coronary sinus. The left innominate vein forms from the left precardinal vein (superior part) and provides the venous drainage towards the right superior vena cava (19). The left precardinal vein later becomes the internal left jugular and left subclavian veins.

PLSVC appears when there are anomalies in the development or anastomosis between the superior precardinal venous systems. Absence or underdevelopment of the innominate vein determines drainage persistence of the left precordial vein into the left common cardinal vein. The left internal jugular and the left subclavian veins drain venous blood through the PLSVC directly into Marshalls' vein and from there into the coronary sinus, which opens into the right atrium.

Information regarding the embryologic development of PLSVC, either on its own or associated with other cardiac malformations is scarce. A study published in 1991 on 351 normally developed human embryos, followed according to the Carnegie development scale, compared the malformation aspects to 1208 human embryos with congenital malformations from the collection of embryological pathology of John Hopkins Hospital (20).

The Carnegie system of embryological development has 23 stages of evolution. According to this, the conclusion was that 
in normally developed human embryos a symmetrical venous system appears in association with the cardiac tube during the Carnegie 9 stage. The relation between the left superior vena cava and the coronary sinus was established in the $12^{\text {th }}$ stage. The functional left superior vena cava is present in the $20^{\text {th }}$ stage, but its lumen is later obliterated by compression between the left atrium and pulmonary hilum. Of the 1208 embryos with congenital anomalies included in that study, $9 \%$ presented PLSVC. There were also frequent associations of PLSVC with atrio-ventricular canal defects, cor triatrium and mitral atresia. The late stage of embryologic development, when the left superior vena cava has its lumen obliterated, suggests its persistence is secondary to cardiac congestion, contributing to blood flow redistribution in a later stage (20).

Several authors have made multiple classifications of cases with this type of venous anomaly (PLSVC). An anatomical classification has been developed regarding the presence or absence of the azygos vein and the innominate anastomosis. Four types were described: the first type is represented by cases presenting an anastomosis between the left and right superior vena cava through the innominate venous trunk; the second type refers to cases presenting completely separated left and right superior vena cava (21); the third type refers to the absence or atrophy of the right superior vena cava, blood drainage being realized through the left superior vena cava, while the fourth case refers to separated left and right superior vena cava, each one presenting its own correspondent azygos vein (21).

Paraclinical examinations (thoracic contrast computed tomography, transthoracic echocardiography), as well as obvious intraoperative presentation, led us to consider our patient as being placed in the $4^{\text {th }}$ category of the previously described classification. We confirmed the absence of the venous anastomosis represented by the innominate trunk due to the fact that the contrast medium injected into the left arm during the computed tomography did not reach the right superior vena cava, instead descending towards the heart through the persistent left vena cava. We also observed the presence of the right azygos vein (on the preoperative computed tomography) as well as of the left azygos vein (intraoperatively). However, transthoracic echocardiography did not identify increased atrial pressure, while computed tomography confirmed the venous drainage into the right atrium.

These data enabled us to conclude that there was no atrial right-to-left shunt, also confirmed by the patients' clinical presentation with no hemodynamic anomalies.

Although rare, PLSVC may present severe associated malformations, requiring further cardiologic investigations (such as transthoracic contrast echocardiography, magnetic resonance imaging or cardiac computed tomography with contrast solution) (22). Transthoracic echocardiography with intravenous saline contrast solution can better indicate the blood flow through the coronary sinus via the left superior vena cava through the presence of microbulae (23). Some authors consider trans-esophageal echocardiography associated with contrast saline solution as being superior to standard transthoracic echocardiography (24). Recent studies have suggested that magnetic resonance imaging is the best means of investigation in the absence of a positive diagnosis, being significantly superior to both transthoracic and transesophageal echocardiography. Magnetic resonance imaging can offer a better view of a much wider field, while using gadolinium contrast makes it far superior to other investigations. Thoracic contrast computed tomography scan can also be useful; however, it should not be omitted that it is an invasive method due to radiation exposure, while iodine contrast solution has its' own limitations.

PLSVC is a very important condition in cardiac surgery, being an absolute contraindication for retrograde cardioplegia (25). Furthermore, PLSVC in association with other cardiac malformations may increase postoperative mortality in children who undergo cardiac surgery, making its identification crucial in preventing intra and postoperative complications (26). Cannulation of PLSVC during surgery depends on several factors: the presence or absence of the innominate vein (between the two venae cava), the absence of the right superior vena cava, its caliber, blood flow through the left superior vena cava as well as the planned surgical procedure (27).

The presence of PLSVC may have important surgical implications, especially during cardiac transplantation, when cardiopulmonary bypass is required (28). Several authors consider that PLSVC may be associated with several cardiac arrhythmias, present in $36 \%$ of cases (29). Several mechanisms responsible for arrhythmia development in patients are represented by: possible histological modifications in the atrioventricular sinus, existence of multiple electric nodes between PLSVC, coronary sinus and right atrium (30), electric generating capacity of the PLSVC which may lead to tachyarrhythmia; interatrial conduction delay or atrial arrhythmia secondary to coronary sinus dilation (31).

The presence of PLSVC is important when the left subclavian vein is used for different catheterization procedures: Swan-Ganz catheterization, usage in renal dialysis (32), in oncological treatment (33) or in cardiac stimulator placement (34-36). In all of the abovementioned situations severe complications may occur: left subclavian vein thrombosis, cardiac arrhythmias, perforation of the coronary sinus, cardiac tamponade, cardiogenic shock or even death.

\section{Conclusion}

Transthoracic echocardiography is mandatory in all cases planned for thoracic or cardiac surgery, as well as in cases 
proposed for different venous catheterizations. In the meantime, the presence of a dilated coronary sinus, observed during echocardiography must serve as a warning for the physician of a possible venous malformation. If PLSVC is suspected, the range of investigations must be broadened in order to confirm it along with any other anatomical malformations; by doing so we may avoid possible severe complications which may arise during the approach of the superior cavae system.

\section{Conflicts of Interest}

There are no conflicts of interest to declare regarding this study.

\section{Authors' Contributions}

CS, CP, AM - performed surgical procedures; RP, IB - prepared the manuscript; IB, CD, NG - data analysis; NG - part of the surgical team; LI, NB - advised about the surgical oncology procedure, revised the final draft of the manuscript.

\section{Acknowledgements}

This work was supported by the project entitled "Multidisciplinary Consortium for Supporting the Research Skills in Diagnosing, Treating and Identifying Predictive Factors of Malignant Gynecologic Disorders", project number PN-III-P1-1.2PCCDI2017-0833.

\section{References}

1 McCotter RE: Three cases of persistent left superior vena cava. Anat Rec 10: 371, 1916.

2 Marshall J: On the development of the great anterior veins in man and mammalia; including an account of certain i-emnants of fetal structure found in the adult, a comparative view of these great occasional peculiarities in the human subject. Philos $\mathrm{Tr}$ Roval Soc 140: 133, 1850.

3 de Leval MR, Ritter DG, McGoon DC and Danielson GK: Anomalous sys-temic venous connection. Surgical considerations. Mayo Clin Proc 50(10): 599-610, 1975. PMID: 1165650.

4 Martinez-Villar M, Gran F, Ferrer Q, Giralt G, Sabate-Rotes A and Albert DC: Persistent left superior vena cava with absent right superior vena cava. Rev Esp Cardiol (Engl Ed) 69(2): 220221, 2016. PMID: 26708343. DOI: 10.1016/j.rec.2015.10.002

5 Biffi M, Bertini M, Ziacchi M, Martignani C, Valzania C, Diemberger I, Bran-zi A and Boriani G: Clinical implications of left superior vena cava persis-tence in candidates for pacemaker or cardioverter-defibrillator implanta-tion. Heart Vessels 24(2): 142-146, 2009. PMID: 19337799. DOI: 10.1007/s00380-0081091-4

6 Perloff JK: Congenital anomalies of vena caval connection. In: The Clinical Recognition of Congenital Heart Disease. 4th ed. Philadelphia: WB Saunders Company, pp. 703-714, 1994.

7 Paval J and Nayak S: A persistent left superior vena cava. Singapore Med J 48(3): e90-e93, 2007. PMID: 17342280.

8 Irwin RB, Greaves M and Schmitt M: Left superior vena cava: revisited. Eur Heart J Cardiovasc Imaging 13(4): 284-291, 2012. PMID: 22301985. DOI: 10.1093/ehjci/jes017
9 Bjerregaard P and Laursen HB: Persistent left superior vena cava. Inci-dence, associated congenital heart defects and frontal plane P-wave axis in a paediatric population with congenital heart disease. Acta Paediatr Scand 69(1): 105-108, 1980. PMID: 7368902. DOI: 10.1111/j.1651-2227.1980.tb07039.x

10 Kanzaki T, Numata S, Yamazaki S and Yaku H: Coronary arteriovenous fis-tula draining into persistent left superior vena cava. Ann Thorac Surg 108(4): e265-e267, 2019. PMID: 30878458. DOI: 10.1016/j.athoracsur.2019.01.082

11 Al Omari MH, Aljarrah QM, Fataftah J, Ghosheh B and Manasara Z: Endo-vascular management of May-Thurner syndrome in a patient with left-sided superior vena cava: A case report. Am J Case Rep 20: 713-718, 2019. PMID: 31104066. DOI: $10.12659 / A J C R .915724$

12 Iimura A, Oguchi T, Shibata M, Matsuo M and Takahashi T: Double superior vena cava and anomaly of cardiovascular system with a review of the literature. Okajimas Folia Anat Jpn 88: 37-42, 2011. PMID: 21882595. DOI: 10.2535/ofaj.88.37

13 Das J, Ray S, Rahman MS and Ghosh J: Axillary lymph node metastasis in gallbladder carcinoma with incidentally detected coexistence of aberrant right subclavian artery with left-sided superior vena cava. Indian J Nucl Med 34(3): 244-246, 2019. PMID: 31293311. DOI: 10.4103/ijnm.IJNM_62_19

14 Kanjwal K, Soos M, Gonzalez-Morales D, Shah I, Madala M, Mughal M and Kang MA: Persistent left superior vena cava and absent right superior vena cava with left subclavian vein stenosis: technical challenges with pacemaker implantation. Case Rep Cardiol 2019: 7271591, 2019. PMID: 31467728. DOI: 10.1155/2019/7271591

15 Blokland D, Lentjes GW, Velthuis BK, Chamuleau SAJ and Rienks R: Per-sistent left superior vena cava draining into the left superior pulmonary vein in a scuba diver: A case report and literature study. Scand J Med Sci Sports 29(8): 1265-1269, 2019. PMID: 31111567. DOI: 10.1111/sms.13471

16 Lembo CM and Latte S: Persistence of the left superior vena cava: a case report. Angiology 35(1): 58-62, 1984. PMID: 6696285. DOI: $10.1177 / 000331978403500108$

17 Ashok G, Deepak A, Sharma MD and Lal G: Ostium primum atrial septal defect with persistent left superior vena cava opening into unroofed coronary sinus - a rare entity. Echocardiography 36(7): 1421-1422, 2019. PMID: 31215689. DOI: 10.1111/echo.14408

18 Sahin T, Kilic T, Celikyurt U, Bildirici U and Ural D: Persistent left superior vena cava and partial anomalous pulmonary venous return in an old asymptomatic female patient. Cardiol Res Pract 2009: 152164, 2009. PMID: 20049316. DOI: 10.4061/ 2009/152164

19 Langman's Medical Embryology. $7^{\text {th }}$ ed. Baltimore: Williams and Wilkins, pp. 221-223, 1995.

20 Nsah EN, Moore GW and Hutchins GM: Pathogenesis of persistent left su-perior vena cava with a coronary sinus connection. Pediatr Pathol 11(2): 261-269, 1991. PMID: 2052508. DOI: $10.3109 / 15513819109064763$

21 Sarodia BD and Stoller JK: Persistent left superior vena cava: case report and literature review. Respir Care 45(4): 411-416, 2000. PMID: 10780037.

22 Marginean C, Marginean CO, Muntean I, Toganel R, Melit LE, Marginean MO and Gozar L: Ultrasonographic features of the persistence of superior left vena cava and pathological cardiac associations in fetus. Case series. Med Ultrason 18(2): 214-217, 2016. PMID: 27239657. DOI: 10.11152/mu.2013.2066.182.fet 
23 Radu S, Floria M, Mitu O and Mitu F: Isolated persistent left superior vein cava unmasked by a simple non-invasive cardiac imaging. Rom J Cardiol 26(2): 168-171, 2016.

24 Horvath SA, Suraci N, D’Mello J and Santana O: Persistent left superior vena cava identified by transesophageal echocardiography. Rev Cardio-vasc Med 20(2): 99-100, 2019. PMID: 31345002. DOI: 10.31083/j.rcm.2019.02.510

25 Vizzardi E, Fracassi F, Farina D, Nardi M, D'Aloia A, Chiari E, Nodari S and Dei CL: Persistence of left superior vena cava, absence of coronary sinus and cerebral ictus. Int $\mathrm{J}$ Cardiol 126(2): e39-e41, 2008. PMID: 18353457. DOI: 10.1016/j.ijcard. 2007.12.077

26 Giuliani-Poncini C, Perez MH, Cotting J, Hurni M, Sekarski N, Pfammatter JP and Di Bernardo S: Persistent left superior vena cava in cardiac con-genital surgery. Pediatr Cardiol 35(1): 7176, 2014. PMID: 23821295. DOI: 10.1007/s00246-013-0743-Z

27 Lenox CC, Zuberbuhler JR, Park SC, Neches WH, Mathews RA, Fricker FJ, Bahnson HT and Siewers RD: Absent right superior vena cava with persistent left superior vena cava: implications and management. Am J Cardiol 45(1): 117-122, 1980. PMID: 7350758. DOI: 10.1016/0002-9149(80)90228-3

28 Boyer R, Sidhu R, Ghandforoush A, Win T and Heidari A: Persistent left superior vena cava: Implications of surgical management. J Investig Med High Impact Case Rep 7: 2324709619855754, 2019. PMID: 31195839. DOI: $10.1177 / 2324709619855754$

29 Bartram U, Van Praagh S, Levine JC, Hines M, Bensky AS and Van Praagh R: Absent right superior vena cava in visceroatrial situs solitus. Am J Car-diol 80(2): 175-183, 1997. PMID: 9230155. DOI: 10.1016/s0002-9149(97)00314-7

30 Maruyama M, Ino T, Miyamoto S, Tadera T, Atarashi $\mathrm{H}$ and Kishida $\mathrm{H}$ : Characteristics of the electrical activity within the persistent left superior vena cava: comparative view with reference to the ligament of Marshall. J Electrocardiol 36(1): 53 57, 2003. PMID: 12607196. DOI: 10.1054/jelc.2003.50004
31 Occhetta E, Dell'Era G, Degiovanni A and Sartori C: Persistence of left su-perior vena cava and focal right atrial tachycardia: Challenges and inter-ventional treatment. Cor et Vasa 57(5): e354-e358, 2015. DOI: 10.1016/j.crvasa.2015.05.008

$32 \mathrm{He} \mathrm{H,} \mathrm{Li} \mathrm{B,} \mathrm{Ma} \mathrm{Y,} \mathrm{Zhang} \mathrm{Y,} \mathrm{Ye} \mathrm{C,} \mathrm{Mei} \mathrm{C,} \mathrm{Yu} \mathrm{S,} \mathrm{Dai} \mathrm{B} \mathrm{and} \mathrm{Liu}$ $\mathrm{Y}$ : Catheteriza-tion in a patient with end-stage renal disease through persistent left supe-rior vena cava: a rare case report and literature review. BMC Nephrol 20(1): 202, 2019. PMID: 31164092. DOI: $10.1186 / \mathrm{s} 12882-019-1339-5$

33 Kang J, Liu B and Sun W: Two successful insertions of peripherally insert-ed central catheters in a patient with persistent left superior vena cava: A case report. Medicine (Baltimore) 98(38): e16988, 2019. PMID: 31567935. DOI: 10.1097/MD.0000000000016988

34 Pyeritz RE and Humphries JO: Partial endocardial cushion defect and persistence of the left superior vena cava draining into the left atrium in a 34-year-old man with features of the Kaufman and Marfan syndromes. Johns Hopkins Med J 146(1): 28-32, 1980. PMID: 7354578.

$35 \mathrm{Li} \mathrm{T}, \mathrm{Xu} \mathrm{Q}$, Liao HT, Asvestas D, Letsas KP and Li Y: Transvenous dual-chamber pacemaker implantation in patients with persistent left superior vena cava. BMC Cardiovasc Disord 19(1): 100, 2019. PMID: 31035937. DOI: 10.1186/s12872-0191082-7

36 Sato T, Ishida J, Kojima $\mathrm{T}$ and Komuro I: Successful transvenous pace-maker implantation via re-directed left superior vena cava. Circ J 83(10): 2082, 2019. PMID: 30930426. DOI: 10.1253/circj.CJ-19-0010
Received December 26, 2019

Revised January 5, 2020

Accepted January 7, 2020 